\title{
The Near-Zone Magnetic Field of a Small Circular-Loop Antenna
}

\author{
Frank M. Greene* \\ Institute for Basic Standards, National Bureau of Standards, Boulder, Colo. 80302
}

(August 21, 1967)

\begin{abstract}
An improved formula is derived for accurately computing the near-zone magnetic field of a small circular transmitting loop antenna. Such a field can serve as a reference standard for calibrating field-strength meters employing small receiving loop antennas in the frequency range $30 \mathrm{~Hz}$ to $30 \mathrm{MHz}$.

This formula includes correction terms for frequency (due to the finite time of propagation), as well as corrections for the finite radii of both the transmitting and receiving loops. Other formulas appearing in the literature often fail to include such corrections which can result in errors of up to 20 percent and more in computing standard-field values.

The NBS formula is derived by expanding the integrand of the retarded vector potential into an infinite series of spherical Hankel functions of increasing order. The resulting series expression is in error by less than 0.2 percent, is rapidly converging and simple to use without recourse to a table of functions or a computer.

Key Words: Loop-antenna field strength, magnetic field-strength standard, mutual inductance of coaxial circular filaments, near-zone magnetic field, transmitting loop antenna.
\end{abstract}

\section{Introduction}

The purpose of this paper is to present a derivation of an improved high-frequency formula intended for use in accurately computing the near-zone magnetic component of the electromagnetic field of a small circular transmitting loop. Such a field can serve as a reference standard for calibrating field-strength meters employing small receiving loop antennas in the frequency range $30 \mathrm{~Hz}$ to $30 \mathrm{MHz}$. The new formula includes correction terms for frequency (due to the finite time of propagation), as well as corrections for the finite radii of both the transmitting and receiving loops. Formulas used by other workers in the field often do not include such corrections. This new formula is a rapidly converging infinite series. It is simple in form and easy to use by workers in the field without recourse to a table of functions, or to a computer.

An earlier formula, which prompted this derivation, has been used during the past 15 or 20 years at the National Bureau of Standards [1]. ${ }^{1}$ It has served as the basis for the NBS calibration service for commercial and military $\mathrm{CW}$ field-strength meters over the above frequency range. This formula was a single-term expression and was first obtained from a detailed numerical analysis of the existing low-frequency formulas, of which there were many in the literature [2]. Most of these were in the form of an infinite series. Many were slowly converging and required the use of at least two or three terms of the series to yield the accuracy

*Radio Standards Engineering Division, National Bureau of Standards, Boulder, Colo. 80302.

1 Figures in brackets indicate the literature references at the end of this paper. achieved in the single term of the earlier NBS formula, over the usual range of the parameters involved.

From the form and symmetry of this single-term expression, it became apparent that it might be the leading term of a rapidly converging infinite-series expression for the magnetic field of the loop. Efforts were then directed toward finding an analytical solution in series form having the earlier formula as its leading term. A number of methods were tried without success. The one that finally achieved the desired result is based on expanding the integrand of the retarded-vector-potential into an infinite series of spherical Hankel functions of increasing order. While this approach must have occurred to other workers in the past, the author has been unable to find any record of a high-frequency derivation in this particular series form in the literature.

All time-varying quantities used in this paper are expressed in terms of their rms values. Rationalized mks units are used throughout.

\section{Calibration and Use of a Small Loop}

The physical arrangement used at NBS for calibrating small receiving loop antennas is shown in figure 1. The standard magnetic field is produced by a small, single-turn, circular transmitting loop of radius, $r_{1}$ meters, in which a current, $I$ amperes, is flowing. The small receiving loop being calibrated has a radius, $r_{2}$ meters, and is positioned coaxially with respect to the transmitting. loop. The two loops are spaced an axial distance, $d$ meters, apart during the calibration. It is assumed that the distance of 


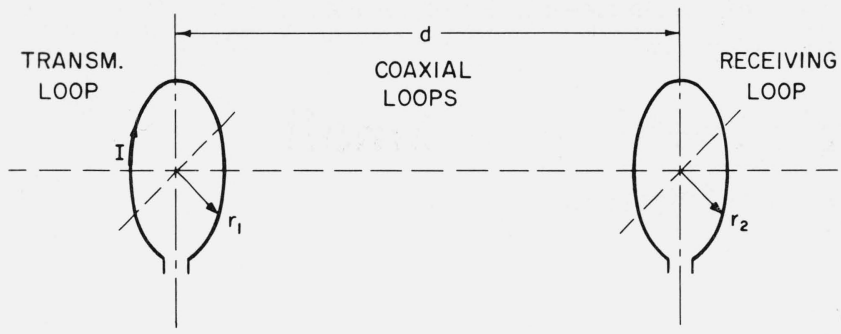

FIgURE 1. Coaxial arrangement of transmitting loop antenna (radius $\mathrm{r}_{1}$ meters), and receiving loop antenna (radius $\mathrm{r}_{2}$ meters), spacing d meters, in the NBS calibration setup.

An RF current, $I$ amperes, flows in the transmitting loop.

separation, $d$, is small compared to the wavelength, $\lambda$, and that near-field conditions prevail.

The response or indication of the field-strength meter is directly proportional to the voltage induced in the receiving loop. The induced voltage is, in turn, proportional to the time-rate of change of the total flux cutting the loop. This is given by Faraday's law of electromagnetic induction, written here for the sinusoidal time-varying case (with the time factor $e^{j \omega t}$ understood),

$$
\oint \bar{E} \cdot \overline{d s}_{2}=-j \omega \iint \bar{B} \cdot \overline{d S}_{2} .
$$

The voltage induced in the loop is given by the lefthand side of eq (1), which is the line integral of the tangential component of the incident electric field, $\bar{E}$, around the periphery, $s_{2}$, of the receiving loop; $\vec{B}$ is the magnetic-flux density at any point on the plane surface, $S_{2}$, bounded by the circular receiving loop; $\omega=2 \pi f$, and $j=\sqrt{-1}$.

When making a field-strength measurement, one does not know the spatial distribution of $\bar{B}$ over the area, $S_{2}$, and it cannot be resolved by the loop. The response can therefore only be expressed in terms of the average field over the area of the loop, and eq (1) becomes

$$
V=-j \omega B_{\mathrm{av}} \cdot S_{2},
$$

where $V$ is the induced voltage and $B_{\text {av }}$ is the normal component of $\bar{B}$ averaged over the area, $S_{2}$, of the receiving loop. This assumes that the relative orientation of the receiving loop with respect to the electricfield vector, $\bar{E}$, is such that the electric-dipole response is negligible [3].

\section{Retarded Vector Potential of a Circular Transmitting Loop}

The basic far-field properties of a small transmitting loop antenna are usually derived for conditions under which second-order corrections to the field due to the finite radii, $r_{1}$ and $r_{2}$, of the transmitting and receiving loops, can be neglected. In near-field use, however, $r_{1}$ and $r_{2}$ are usually not negligible compared to the distance of separation, $d$, between the loops. Therefore, corresponding corrections must be provided in the field magnitudes involved by including both of these radii in the derivation.

The expression for the axial magnetic component of the near field of the electrically-small $\left(2 \pi r_{1} \ll \lambda\right)$ transmitting loop antenna will now be derived. It will be assumed for the present, that the loop current is constant in amplitude and phase around the loop, and that the radius of the wire with which the loop is wound is negligible compared to the loop radius, $r_{1}$, itself. The effect of a non-uniform current distribution on the accuracy of the resulting field formulas at frequencies above 5 or $10 \mathrm{MHz}$ will be discussed later in section 5 . The effect of the finite time of propagation will be included in the derivation.

The magnetic-flux density, $\bar{B}$, at any point, $P$, on the surface, $S_{2}$, of the receiving loop, is given by the curl of the retarded vector potential, $\bar{A}$, at the same point [4], i.e.,

$$
\bar{B}=\nabla \times \bar{A} .
$$

The geometry involved is shown in figure 2 . The average value of the normal component of $\bar{B}$ will be given by

$$
B_{\mathrm{av}}=\frac{1}{S_{2}} \iint \nabla \times \bar{A} \cdot \overline{d S}_{2},
$$

where [4]

$$
\bar{A}=\frac{\mu I}{4 \pi} \int \frac{e^{-j \beta R}}{R} \overline{d s}_{1},
$$

and

$\mu=$ permeability of free-space $\left(\mu=4 \pi \cdot 10^{-7}\right.$, henry/ meter)

$I=$ transmitting-loop current, rms amperes

$\beta=$ wavelength constant $(\beta=2 \pi / \lambda)$

$\lambda=$ free-space wavelength, meters

$f=$ frequency, hertz

$j=\sqrt{-1}$.

It can easily be shown that the vector potential will have only a component, $A_{\phi}$, which is azimuthal at the point, $P$. Let $P$ be chosen arbitrarily to lie in the $x z$ plane where $\phi=0$. Equidistant line elements of length $d s_{1}$ at $+\phi$ and $-\phi$ are paired. These elements can each be resolved into a component, $d s_{1} \sin \phi$, parallel to the $x z$ plane and a component, $d s_{1} \cos \phi$, normal to the $x z$ plane. In taking the resultant, the former components will cancel, while the latter (azimuthal with respect to the point $P$ ) will add. Equation (5) can therefore be rewritten [5], letting $d s_{1}=r_{1} d \phi$,

$$
A_{\phi}=\frac{\mu I r_{1}}{2 \pi} \int_{0}^{\pi} \frac{e^{-j \beta R}}{R} \cos \phi d \phi .
$$


By Stokes's Theorem [6], the integral of the normal component of the curl of $\bar{A}$ over the surface $S_{2}$ bounded by the receiving loop can be transformed into the line integral of the tangential component of $\bar{A}$ around its periphery, i.e.,

$$
\iint \nabla \times \bar{A} \cdot \overline{d S}_{2}=\oint A_{\phi}(P) d s_{2},
$$

where $A_{\phi}(P)$ is the value of the vector potential at the point, $P$, now assumed to lie on the periphery of the receiving loop of radius, $\rho=r_{2}$. Substituting eq (7) in eq (4) gives

$$
B_{\mathrm{av}}=\frac{1}{S_{2}} \oint A_{\phi}(P) d s_{2} .
$$

Since the receiving and transmitting loops are coaxial, it can be seen from the circular symmetry involved, that $A_{\phi}(P)$ will be constant around the periphery of the receiving loop, so that eq (8) becomes simply

$$
B_{\mathrm{av}}=\frac{2 \pi r_{2}}{S_{2}} A_{\phi}(P) \text {. }
$$

Substituting eq (6) in eq (9), letting $B_{\mathrm{av}}=\dot{\mu} H_{\mathrm{av}}$ and $S_{2}=\pi r_{2}^{2}$, gives

$$
H_{\mathrm{av}}=\frac{I r_{1}}{\pi r_{2}} \int_{0}^{\pi} \frac{e^{-j \beta R}}{R} \cos \phi d \phi
$$

where now

$$
R=\left[d^{2}+r_{1}^{2}+r_{2}^{2}-2 r_{1} r_{2} \cos \phi\right]^{1 / 2},
$$

as can be determined from figure 2. $H_{\mathrm{av}}$ is the average normal component of magnetic field strength over the area of the circular receiving loop.

\section{Evaluation of the Complex Integral}

The problem, now, is to evaluate the integral in eq (10). Maxwell [7] was the first to accomplish this for the static case $(\beta=0)$, using elliptic integrals which can be expressed in several series forms [8]. Numerous other workers have since evaluated this integral in various ways for the static case. Literally, over onehundred different series expressions appear in the literature [9] for the mutual inductance between coaxial circular filaments, to which the present problem is directly related.

To the above will now be added still another series solution, this time also including the effect of retarded time. Equation (10) will be evaluated using the relationship [10]

$$
\frac{e^{-j \beta R}}{-j \beta R} \equiv h_{0}^{(2)}(\beta R)
$$

where $h_{0}^{(2)}(\beta R)$ is the zero-order spherical Hankel function of the second kind. Such a function is often used to represent an outward-traveling (+) wave,

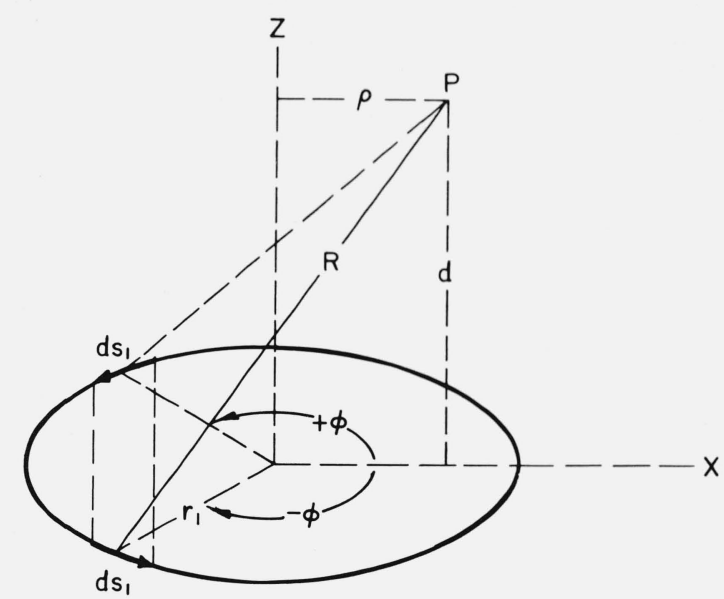

FIGURE 2. Geometry used for determining the vector potential and near-zone magnetic field of a small circular transmitting loop.

The loop lies in a plane normal to the $x z$ plane with its center at the origin of coordinates.

whereas a similar function of the first kind, $h_{0}^{(1)}(\beta R)$, would have been used to represent an inward-traveling (-) wave. The function, $h_{0}^{(2)}(\beta R)$, will now be expanded into an infinite series of higher order spherical Hankel functions. These will be substituted back into eq (10) and integrated term by term.

The expansion can be accomplished using the following relationship based on Erdélyi [11],

$$
h_{0}^{(2)}(\beta \boldsymbol{R})=h_{0}^{(2)}(\sqrt{u+v})=\sum_{m=0}^{\infty} \frac{1}{m !}\left[\frac{-v}{2 \sqrt{u}}\right]^{m} h_{m}^{(2)}(\sqrt{u}),
$$

where $m$ is an integer. This series converges when $|u|>|v|$. Let

$$
\sqrt{u+v} \equiv \beta\left(R_{0}^{2}-2 r_{1} r_{2} \cos \phi\right)^{1 / 2},
$$

where

$$
R_{0}^{2}=d^{2}+r_{1}^{2}+r_{2}^{2} .
$$

Let

$$
u=\beta^{2} R_{0}^{2}
$$

and

$$
v=-\beta^{2}\left(2 r_{1} r_{2} \cos \phi\right) .
$$

Substituting eq (16) and eq (17) in eq (13) gives

$$
h_{0}^{(2)}(\beta R)=\sum_{m=0}^{\infty} \frac{1}{m !}\left[\beta \frac{r_{1} r_{2}}{R_{0}}\right]^{m} h_{m}^{(2)}\left(\beta R_{0}\right) \cos ^{m} \phi .
$$

Substituting eq (18) back into eq (10) gives

$H_{\mathrm{av}}=-j \frac{\beta I r_{1}}{\pi r_{2}} \int_{0}^{\pi} \sum_{m=0}^{\infty} \frac{1}{m !}\left[\beta \frac{r_{1} r_{2}}{R_{0}}\right]^{m} h_{m}^{(2)}\left(\beta R_{0}\right) \cos ^{m+1} \phi d \phi$. 
The infinite series in eq (19) will converge [11] when $R_{0}^{2}>2 r_{1} r_{2}$ if $\phi=0$ or if $\phi=\pi$. Furthermore it can be shown [12] using, for example, the Weierstrass $M$ Test, that eq (19) will converge uniformly in the interval $0 \leqslant \phi \leqslant \pi$. Equation (19) can then be integrated term by term, yielding

$H_{\mathrm{av}}=-j \frac{\beta I r_{1}}{\pi r_{2}} \sum_{m=0}^{\infty} \frac{1}{m !}\left[\beta \frac{r_{1} r_{2}}{R_{0}}\right]^{m} h_{m}^{(2)}\left(\beta R_{0}\right) \int_{0}^{\pi} \cos ^{m+1} \phi d \phi$.

The integral in eq (20) can be evaluated from the following relations [13]:

$$
\begin{array}{lr}
\int_{0}^{\pi} \cos ^{\prime \prime} \phi d \phi=0, & \text { (if } p \text { is odd) } \\
(21)
\end{array}
$$

When these results are substituted into eq (20), with an appropriate change in the index of summation, eq (20) becomes

$$
\left.\left.H_{\mathrm{av}}=-j \frac{\beta I r_{1}}{r_{2}} \sum_{m=0}^{\infty} \frac{1}{(2 m+1) ! 2 \cdot 4 \cdot 6 \ldots 2 m+2} \frac{1 \cdot 3 \cdot 5 \ldots 2 m+1}{R_{0}}\right]^{\beta r_{1} r_{2}}\right]^{2 m+1} h_{2 m+1}^{(2)}\left(\beta R_{0}\right) .
$$

The spherical Hankel functions in eq (23) can now be changed into their exponential polynomial forms as given by eq (All) to eq (Al6) in the Appendix. When this is done, eq (23) can be written

$$
\begin{aligned}
H_{\mathrm{av}}= & \frac{I S_{1}}{2 \pi R_{0}^{3}}\left\{\left[1+j \beta R_{0}\right]+\frac{15}{8}\left(\frac{r_{1} r_{2}}{R_{0}^{2}}\right)^{2}\left[1+j \beta R_{0}-\frac{6}{15} \beta^{2} R_{0}^{2}-j \frac{1}{15} \beta^{3} R_{0}^{3}\right]\right. \\
& +\frac{315}{64}\left(\frac{r_{1} r_{2}}{R_{0}^{2}}\right)^{4}\left[1+j \beta R_{0}-\frac{420}{945} \beta^{2} R_{0}^{2}-j \frac{105}{945} \beta^{3} R_{0}^{3}+\frac{15}{945} \beta^{4} R_{0}^{4}+j \frac{1}{945} \beta^{5} R_{0}^{5}\right] \\
& +\ldots\} e^{-j \beta R_{0}}, \quad \text { amperes/meter. }
\end{aligned}
$$

Under the conditions that $\beta R_{0} \leqslant 1.0$, and $r_{1} r_{2} / R_{0}^{2}$ $\leqslant 1 / 16$, terms in $\beta R_{0}$ higher than the first power can be ignored without introducing an error in eq (24) of more than 0.2 percent. Therefore, if the term $\left(1+j \beta R_{0}\right)$ is factored out of eq (24), the magnitude of eq (24) can be written as

$$
\left|H_{\mathrm{av}}\right| \cong \frac{I S_{1}}{2 \pi R_{0}^{3}}\left[1+\frac{15}{8}\left(\frac{r_{1} r_{2}}{R_{0}^{2}}\right)^{2}+\frac{315}{64}\left(\frac{r_{1} r_{2}}{R_{0}^{2}}\right)^{4}+\ldots\right]\left(1+\beta^{2} R_{0}^{2}\right)^{1 / 2} .
$$

This is the desired expression for the normal component of the magnetic field averaged over the area, $S_{2}$, of the receiving loop.

The effect of the neglected terms in $\beta R_{0}$ on the accuracy of eq (25) decreases rapidly as $\beta R_{0}$ is progressively decreased below 1.0 , other factors remaining 
the same. At the lower frequencies where corrections due to $\beta R_{0}$ can be neglected entirely $\left(\beta R_{0} \ll 1\right)$, the mathematical accuracy of eq (25) is limited only by the number of terms used in the infinite series.

For the conditions previously given, the first correction term in the infinite series of eq (25) will contribute less than 1 percent, so that for this case eq (25) can be further simplified to

$$
\left|H_{\mathrm{av}}\right| \cong \frac{I S_{1}}{2 \pi R_{0}^{3}}\left(1+\beta^{2} R_{0}^{2}\right)^{1 / 2}, \text { amperes/meter. }
$$

It should be noted that eq (26) is essentially identical to the expression for the axial component of the magnetic field of a classical, infinitesimal magnetic dipole. The difference is that the distance factor, $R_{0}$, in eq (26) includes correction terms for the finite radii, $r_{1}$ and $r_{2}$, of the transmitting and receiving loops, respectively, as given by eq (15).

As previously stated, the response of a field-strength meter employing a small-loop antenna is directly proportional to the average normal component of the magnetic field strength, $H_{\mathrm{av}}$, incident on the loop. However, for many years it has been customary to express the calibration of such an instrument in terms of the equivalent electric field strength, $E$, that would be associated with $H$ for the case of a uniform plane wave (where $E \cong 120 \pi H$ ). This relationship, when substituted in eq (26) with $S_{1}=\pi r_{1}^{2}$, gives

$$
\left|E_{\mathrm{av}}\right| \cong \frac{60 \pi r_{1}^{2} I}{R_{0}^{3}}\left(1+\beta^{2} R_{0}^{2}\right)^{1 / 2}, \quad \text { volts/meter. }
$$

Needless to say, this manner of expressing the response will be valid only when the field-strength meter is used to measure a uniform plane wave. The measurement will be of questionable value for all cases in which the local $|E / H|$ ratio departs from the free-space value [14].

Standard-field formulas appearing in the literature [15] to [17] often fail to correct for the finite radii of the transmitting or receiving loop antennas, or for frequency. It is of interest to evaluate the errors resulting from the failure to apply these corrections, all of which are included in the formulas derived in this paper.

Failure to correct for the radius, $r_{2}$, of the receiving loop will result in an error of from 1.0 percent (for $d=1.25 \mathrm{~m}, r_{2}=0.1 \mathrm{~m}$ ), to 20 percent (when $d=0.5 \mathrm{~m}$, $r_{2}=0.2 \mathrm{~m}$ ). Failure to simultaneously correct for the radius, $r_{1}$, of the transmittin $\%$ loop will approximately double these errors.

The value of field strength given by eq (25) to eq (27) is essentially independent of frequency at the lower frequencies (where $\beta R_{0} \ll 1$ ). Failure to apply the frequency correction indicated, at the higher frequencies, will introduce an error of, e.g., 1.0 percent at $5 \mathrm{MHz}$, which increases to approximately 27 percent at $30 \mathrm{MHz}$ (when $d=1.25 \mathrm{~m}, r_{1}=r_{2}=0.1 \mathrm{~m}$ ). If the transmitting loop circumference exceeds approximately $\lambda / 16$, the effect of a nonuniform current

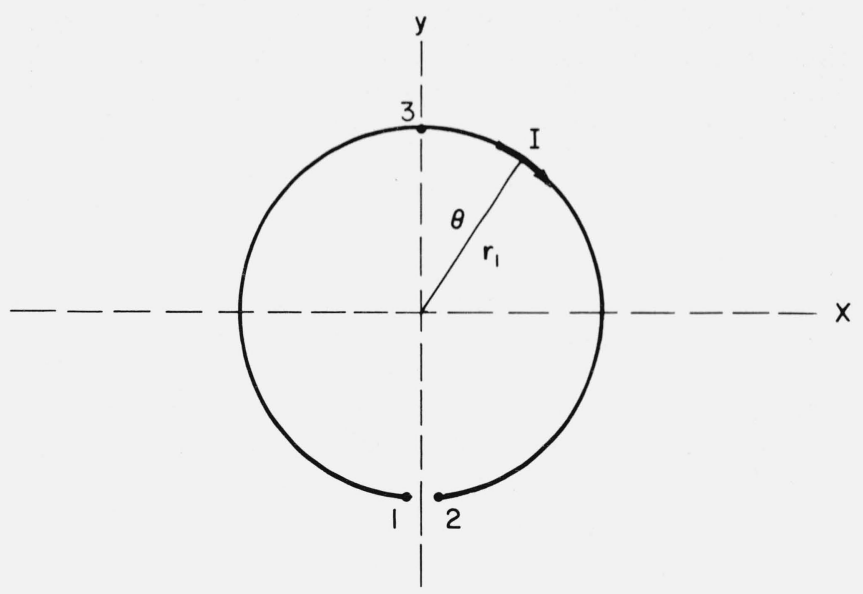

FIGURE 3. Geometry used to analyze the current distribution on a circular transmitting loop antenna.

The loop is treated as a circular balanced transmission line, fed at points 1,2 , and shortcircuited at the receiving end (point 3 ). Distance along the loop periphery, $l=\theta r_{\|}$meters, is measured from point 3 .

distribution will also have to be taken into account, as discussed in the next section.

\section{Loop-Antenna Current Distribution}

In the preceding derivation, the loop current distribution was assumed, for simplicity, to be constant in amplitude and phase around the loop. Actually, a standing wave of current exists on the loop, and the assumption of a constant current will not be valid unless the loop is small electrically (i.e., unless $\left.2 \pi r_{1} \ll \lambda\right)$.

The complete solution to this problem for an arbitrary current distribution [18] is quite complex. However, the effect of a nonuniform current distribution at frequencies from roughly 5 to $30 \mathrm{MHz}$ can be determined from the following approximate analysis. The single-turn loop can be considered as a circular, balanced transmission line fed at points 1,2 , as indicated in figure 3 , and short-circuited at its receiving end (point 3).

If the radius of the wire with which the loop is wound is small compared to the loop radius, $r_{1}$, it can be assumed that the current distribution around the loop is, to a first approximation, of the hyperbolic-cosine form [19],

$$
I \cong I_{0} \cosh \gamma l
$$

where

$I_{0}=$ reference current at point 3 (fig. 3 )

$\gamma=$ propagation constant $=\alpha+j \beta$

$\alpha=$ attenuation constant, nepers/meter

$\beta=$ wavelength constant $=2 \pi / \lambda$, radians per meter

$\lambda=$ free-space wavelength, meters

$l=$ peripheral distance, in meters, measured from point 3 . 
The loop will be considered sufficiently loss free that $\alpha$ can be assumed negligible compared to $\beta$. This is a reasonable assumption for the type of loop used, so that eq (28) can be written

$$
I \cong I_{0} \cosh (j \beta l) \cong I_{0} \cos \beta l .
$$

As can be seen from eq (29) the current will have a constant phase around the loop for this case.

For the size loop of interest here, the magnitude of the magnetic field strength given by eq (24) to eq (26) will be, to a first approximation, proportional to the average value of the current around the transmitting loop. The average current is given by

$$
I_{\mathrm{av}}=\frac{I_{0}}{2 l_{1}} \int_{-l_{1}}^{+l_{1}} \cos \beta l d l=I_{0} \frac{\sin \beta l_{1}}{\beta l_{1}},
$$

where $l_{1}=$ half the loop circumference, meters and $l=\theta r_{1}$ meters from figure 3 .

TABlE 1. Percent difference between $\mathrm{I}_{\mathrm{av}}$ and $\mathrm{I}_{0}$

\begin{tabular}{c|c}
\hline \hline$\frac{2 \pi r_{1}}{\lambda}$ & $\left(1-\frac{I_{\mathrm{av}}}{I_{0}}\right) \cdot 100$ \\
$\begin{array}{c}\text { Loop } \\
\text { circumference, } \\
\text { wavelengths }\end{array}$ & $\begin{array}{c}\text { Current } \\
\text { difference, } \\
\text { percent }\end{array}$ \\
\hline $1 / 2$ & 36 \\
$1 / 4$ & 10 \\
$1 / 8$ & 2.6 \\
$1 / 16$ & 0.64 \\
$1 / 32$ & .16 \\
\hline
\end{tabular}

Table 1, which is based on eq (30), shows the approximate percentage difference between $I_{\text {av }}$ and $I_{0}$ for several sizes of low-loss loops $(\alpha \ll \beta)$, with their circumferences expressed in fractions of a wavelength, $2 \pi r_{1} / \lambda$. As can be seen, the correction due to the nonuniform current distribution will be less than 1.0 percent at the highest operating frequency, provided the loop circumference does not exceed $\lambda / 16$. This correction decreases rapidly with decreasing frequency, becoming less than 0.2 percent at frequencies below $15 \mathrm{MHz}$ for a loop having a radius $r_{1}=0.1 \mathrm{~m}$.

These corrections, based on an assumed cosinusoidal current distribution around the loop, are in quite close agreement with similar corrections based on a recent theoretical analysis by T. T. Wu [20]. The difference in the correction values obtained by the two methods in less than 0.02 percent for the conditions involved here. It is interesting to note that for this case the loop current distribution obtained by $\mathrm{Wu}$ reduces essentially to the cosine form given by eq (29) above. This is shown in figure 4 for a loop having a radius $r_{1}=0.1 \mathrm{~m}$ operating at a frequency $f=30 \mathrm{MHz}$.

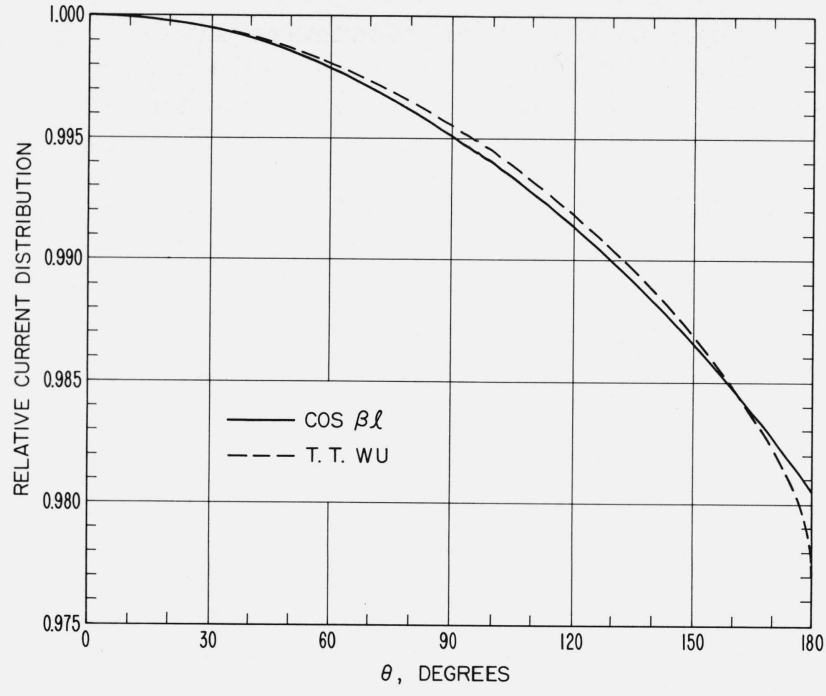

FIGURE 4. Relative current distribution around a small circular transmitting loop antenna having a radius $\mathrm{r}_{1}=0.1 \mathrm{~m}$, for a frequency $\mathrm{f}=30 \mathrm{MHz}$.

The current distribution, based on an analysis by T. T. $\mathrm{Wu}_{\mathrm{u}}[20]$, is compared with an assumed cosine distribution determined from eq $(29)$ in the text, where $l=\theta r_{1}$ from figure 3 . A wire radius $a=0.001 \mathrm{~m}$ was used in the $\mathrm{Wu}$ analysis.

\section{An Alternate Method for Evaluating the Integral}

The infinite series of eq (25) can be easily verified for the static case $(\beta=0)$ using a much simpler method. This is done by rewriting eq (10) in the form

$$
H_{\mathrm{av}}=\frac{I r_{1}}{\pi R_{0} r_{2}} \int_{0}^{\pi} F(\phi) \cos \phi d \phi,
$$

where

$$
F(\phi) \equiv\left(1-\frac{2 r_{1} r_{2} \cos \phi}{R_{0}^{2}}\right)^{-1 / 2}
$$

The binomial expansion of eq (32) is then substituted back into eq (31) and the result integrated term by term, yielding the infinite series of eq (25) directly, provided $R_{0}^{2}>2 r_{1} r_{2}$ as before. The first few terms of the binomial expansion of eq (32) are as follows:

$$
\begin{aligned}
F(\phi)=1 & +\left(\frac{r_{1} r_{2}}{R_{0}^{2}}\right) \cos \phi+\frac{3}{2}\left(\frac{r_{1} r_{2}}{R_{0}^{2}}\right)^{2} \cos ^{2} \phi \\
+ & \frac{5}{2}\left(\frac{r_{1} r_{2}}{R_{0}^{2}}\right)^{3} \cos ^{3} \phi+\frac{35}{8}\left(\frac{r_{1} r_{2}}{R_{0}^{2}}\right)^{4} \cos ^{4} \phi \\
& +\frac{63}{8}\left(\frac{r_{1} r_{2}}{R_{0}^{2}}\right)^{5} \cos ^{5} \phi+\ldots .
\end{aligned}
$$

Substituting eq (33) back into eq (31) and performing the required integration, making use of eq (21) and eq (22), yields for the static case 
$H_{\mathrm{av}} \cong \frac{I S_{1}}{2 \pi R_{0}^{3}}\left[1+\frac{15}{8}\left(\frac{r_{1} r_{2}}{R_{0}^{2}}\right)^{2}+\frac{315}{64}\left(\frac{r_{1} r_{2}}{R_{0}^{2}}\right)^{4}+\ldots.\right]$

which is identical to the infinite series in eq (25).

\section{Summary and Conclusions}

An improved high-frequency formula has been derived for accurately computing the near-zone magnetic field of a small $\left(2 \pi r_{1} \ll \lambda\right)$, circular, transmitting loop antenna. Such a field is used as a reference standard at NBS for the calibration of field-strength meters [1], [14] employing small receiving loop antennas in the frequency range $30 \mathrm{~Hz}$ to $30 \mathrm{MHz}$.

The new formula is in the form of an infinite series and includes correction terms for frequency (due to the finite time of propagation), as well as corrections for the finite radii of both the transmitting and receiving loops. The derivation is based on a current of constant amplitude and phase around the transmitting loop. However, the effect of a nonuniform current distribution at frequencies above 5 or $10 \mathrm{MHz}$ is also analyzed. Formulas used by other workers in the field [15] to [17] often fail to include these refinements which can result in errors of up to 20 percent and more in computing standard-field values.

The accuracy of the new formula is basically limited only by the number of terms used in the series. At the highest frequency normally employed, $30 \mathrm{MHz}$, the error in one of the approximate forms given does not exceed 0.2 percent for the conditions stated and decreases rapidly with decreasing frequency. The series expression is rapidly converging and simple to use by workers in the field without recourse to a table of functions or a computer.

The author thanks William H. Lupton (formerly with the NBS Radio Standards Laboratory) as well as Otto N. Strand and others (formerly with the NBS mathematics group) for their assistance and helpful suggestions relating to this problem. The author also acknowledges with thanks the assistance of Ezra B. Larsen who made a detailed numerical analysis of T. T. Wu's work which was used in the preparation of figure 4 .

\section{Appendix}

For the convenience of the reader, explicit expressions for the various Bessel functions used herein, but not commonly found in compilations, are listed below.

The spherical Hankel functions of the second kind, used in this paper, are defined in terms of Bessel functions of the first and second kinds, $J_{\nu}(x)$ and $N_{\nu}(x)$ respectively, by the relation [21]

$$
h_{n}^{(2)}(x)=\sqrt{\frac{\pi}{2 x}}\left(J_{n+1 / 2}(x)-j N_{n+1 / 2}(x)\right),
$$

Besisel functions of odd half order have particularly simple forms in terms of trignometric functions [22]. A few of these relationships are as follows:

$$
\begin{aligned}
& J_{1 / 2}(x)=\sqrt{\frac{2}{\pi x}} \sin x \\
& J_{3 / 2}(x)=\sqrt{\frac{2}{\pi x}}\left(\frac{\sin x}{x}-\cos x\right) \\
& J_{5 / 2}(x)=\sqrt{\frac{2}{\pi x}}\left(\left[\frac{3}{x^{2}}-1\right] \sin x-\frac{3}{x} \cos x\right) \\
& N_{1 / 2}(x)=\sqrt{\frac{2}{\pi x}}(-\cos x) \\
& N_{3 / 2}(x)=\sqrt{\frac{2}{\pi x}}\left(-\sin x-\frac{\cos x}{x}\right) \\
& N_{5 / 2}(x)=\sqrt{\frac{2}{\pi x}}\left(-\frac{3}{x} \sin x-\left[\frac{3}{x^{2}}-1\right] \cos x\right) .
\end{aligned}
$$

The recurrence relations for calculating these functions for higher orders are [23]

$$
\begin{aligned}
& J_{n+1}(x)=\frac{2 n}{x} J_{n}(x)-J_{n-1}(x) \\
& N_{n+1}(x)=\frac{2 n}{x} N_{n}(x)-N_{n-1}(x) .
\end{aligned}
$$

If these two relations are substituted into the definition of the spherical Hankel functions, given by eq (Al) of this appendix, the recurrence relation for spherical Hankel functions is obtained as [24]

$$
h_{n+1}(x)=\frac{2 n+1}{x} h_{n}(x)-h_{n-1}(x) .
$$

Using the above relations several spherical Hankel functions of higher order can be determined.

$$
\begin{aligned}
& h_{0}^{(2)}(x)=\frac{e^{-j x}}{x}(+j) \\
& h_{1}^{(2)}(x)=\frac{e^{-j x}}{x}\left(-1+\frac{j}{x}\right) \\
& h_{2}^{(2)}(x)=\frac{e^{-j x}}{x}\left(-j-\frac{3}{x}+j \frac{3}{x^{2}}\right) \\
& h_{3}^{(2)}(x)=\frac{e^{-j x}}{x}\left(1-j \frac{6}{x}-\frac{15}{x^{2}}+j \frac{15}{x^{3}}\right)
\end{aligned}
$$


$h_{4}^{(2)}(x)=\frac{e^{-j x}}{x}\left(+j+\frac{10}{x}-j \frac{45}{x^{2}}-\frac{105}{x^{3}}+j \frac{105}{x^{4}}\right)$

$h_{5}^{(2)}(x)=\frac{e^{-j x}}{x}\left(-1+j \frac{15}{x}+\frac{105}{x^{2}}-j \frac{420}{x^{3}}-\frac{945}{x^{4}}+j \frac{945}{x^{5}}\right)$.

It should be noted that the expression for each of the above functions is exact, each being a polynomial of degree $(n+1)$. These Hankel functions can also be derived somewhat more directly from the following relation based on Erdélyi [25]:

$$
h_{n}^{(2)}(x)=j(-1)^{n} x^{n}\left(\frac{d}{x d x}\right)^{n} \frac{e^{-j x}}{x} .
$$

\section{References}

[1] F. M. Greene, Calibration of commercial radio field-strength meters at the National Bureau of Standards, NBS Circular 517 (Dec. 1951)

[2] E. B. Rosa and F. W. Grover, Formulas and tables for the calculation of mutual and self-inductance, Bulletin of the Bureau of Standards 8, pp. 1-238 (Jan. 1912).

[3] H. Whiteside and R. W. P. King, The loop antenna as a probe, IEEE Trans. on Antennas and Propagation AP-12, pp. 291-297 (May 1964).

[4] S. Ramo, J. R. Whinnery, and T. Van Duzer, Fields and Waves in Communication Electronics, p. 264 and p. 643 (John Wiley \& Sons, Inc., New York, N.Y., 1965).

[5] W. R. Smythe, Static and Dynamic Electricity, pp. 270-271 (McGraw-Hill Book Co., Inc., New York, N.Y., 1950).

[6] S. Ramo, Op. Cit., pp. 118-119.

[7] J. C. Maxwell, A Treatise on Electricity and Magnetism, Vol. II, Sec. 701, pp. 338-340 (Oxford University Press, London, Third Edition, 1892).
[8] E. B. Rosa, Op. Cit., Sec. 1, pp. 6-20.

[9] F. W. Grover, Inductance Calculations (working formulas and tables), pp. 77-78 (D. Van Nostrand \& Co., Inc., New York, N.Y., 1946).

[10] R. F. Harrington. Time-Harmonic Electromagnetic Fields. pp. 264-269 and pp. 460-464 (McGraw-Hill Book Co., Inc., New York, N.Y., 1961).

[11] A. Erdélyi, Higher Transcendental Functions, Vol. II, formulas 12 and 13 or formula 18, p. 100 (McGraw-Hill Book Co., Inc., New York, N.Y., 1953).

[12] I. S. and E. S. Sokolnikoff, Higher Mathematics for Engineers and Physicists, pp. 25-28 (McGraw-Hill Book Co.. Inc., New York, N.Y., 1941).

[13] H. B. Dwight, Tables of Integrals and Other Mathematical Data, formula 858.44, p. 215 (The MacMillan Company, New York, N.Y., Fourth Edition, 1961).

[14] F. M. Greene, NBS field-strength standards and measurements $(30 \mathrm{~Hz}$ to $1000 \mathrm{MHz}$ ), Proc. IEEE 55, No. 6, pp. 970-981 (June 1967).

[15] W. O. Swinyard, Measurement of loop-antenna receivers, Proc. IRE 29, No. 7, pp. 382-387 (July 1941).

[16] G. A. Morgan, Jr., Analysis and calibration of loop probes, Report R-3486, Naval Research Laboratory, Washington, D.C. (June 17, 1949).

[17] The Radio-Frequency Interference Meter, Bureau of Ships Publication NAVSHIPS 94180, pp. 108-115 (Navy Department, Washington 25, D.C., July 1962).

[18] E. J. Martin, Jr., Exact expressions for the vector potential produced by circular loop antennas, Proc. IEEE (Correspondence) 51, pp. 1042-1043 (July 1963).

[19] G. Glinski, Note on circular loop antennas with non-uniform current distribution, J. Applied Phys. 18, pp. 638-644 (July 1947).

[20] T. T. Wu, Theory of the thin circular loop antenna, J. Math. Phys. 3, No. 6, pp. 1301-1304 (Nov.-Dec. 1962).

[21] M. Abramowitz and I. A. Stegun, Handbook of Mathematical Functions, National Bureau of Standards, AMS-55, formulae 10.1.1, p. 437 (1964).

[22] H. B. Dwight, Op. Cit., formulas 809.01-809.25, pp. 193-194

[23] H. B. Dwight, Op. Cit., formula 801.3, p. 187, and formula 802.3 , p. 188.

[24] M. Abramowitz, Op. Cit., formula 10.1.19, p. 439.

[25] A. Erdélyi, Op. Cit., formula 10, p. 78.

(Paper 71C4-263) 